\title{
Increased incidence of massive hemorrhage at uncommon sites after initiation of systemic anticoagulation in critically ill patients with coronavirus disease 2019 (COVID-19) infection
}

\author{
Salwa A. Koubaissi ${ }^{1}$. Michella Abi Zeid Daou ${ }^{1} \cdot$ Rayan Mohamad $^{1}$ - Ahmad Husari ${ }^{1}$ (D) \\ Accepted: 22 April 2021 / Published online: 28 May 2021 \\ ( ) The Author(s), under exclusive licence to Springer Science+Business Media, LLC, part of Springer Nature 2021
}

\begin{abstract}
Background The management of the Coronavirus disease 2019 (COVID-19) infected patients continues to be challenging. Critically ill COVID patients are at increased risk of serious thrombotic events and hence increased mortality. On the other side, COVID-19 patients are also showing major life-threatening bleeds, especially when systemic anticoagulation is used. Pro-coagulant propensity in critically ill COVID-19 patients have been published, but very few have described the incidence of major bleeding and its characteristics.

Methods In this study, we retrospectively observed the incidence of major bleed in 25 critically ill COVID-19 patients admitted to the Intensive Care Unit at the American University of Beirut Medical Center. Six cases were identified and described together with their outcome.

Results Major bleeding occurred in six of the 25 studied patients. Four patients were on therapeutic anticoagulation at the onset of the bleed, two required embolization for bleeding control and one died from hemorrhagic shock. Half of the described cases had unusual sites of bleeding including gluteal and abdominal wall muscles.

Conclusions A high rate of major bleeding was witnessed in our sample of critically ill patients with COVID-19 infection, with the majority being on therapeutic anticoagulation. This rate may be higher than previously reported, necessitating additional attention from the treating physician when considering empiric therapeutic anticoagulation. Moreover, the uncommon sites of bleeding shed the light on the need for additional studies in our population to identify the predisposing risk factors and mechanisms behind it.
\end{abstract}

Keywords Hemorrhage · Thromboembolism · Coronavirus · Critical illness · Heparin

Ahmad Husari

ah51@aub.edu.lb

Salwa A. Koubaissi

sk162@aub.edu.lb

Michella Abi Zeid Daou

ma379@aub.edu.lb

Rayan Mohamad

rm153@aub.edu.lb

1 Pulmonary and Critical Care Division, Department of Internal Medicine, American University of Beirut Medical Center, Riad El-Solh, P.O. Box 11-236, Beirut 1107 2020,

Lebanon

\section{Introduction}

The management of patients with the Coronavirus disease 2019 (COVID-19) infection is challenged by significant multiorgan dysfunction and disruption of normal hemostasis [1]. In particular, COVID-19 critically ill patients are more likely to develop a systemic procoagulant state with elevated D-dimer levels and microvascular injury leading to thrombus formation and pulmonary embolism [2]. It is, therefore, recommended that $\mathrm{D}$-dimer and fibrinogen levels be monitored, and all hospitalized COVID-19 patients to receive thromboembolism prophylaxis [1,3]. On the other hand, it is emerging that COVID-19 patients are more prone to develop major life-threatening bleeds when systemic anticoagulation is initiated [4-6]. Major bleed is defined as bleeding leading to direct death, an acute hemoglobin [Hb] level drop of $2 \mathrm{~g} / \mathrm{dL}$ or more, and or acute transfusions of two or 
more units of packed red blood cells [7]. The risk of major bleeding in the general critically ill population receiving thromboprophylaxis, independently of the type of pharmacologic thromboprophylaxis used, has been estimated to be 5.6\% [8]. Many reports have highlighted the increased risk of venous thromboembolism (VTE) in critically ill patients with COVID-19 when compared to the general population $[6,9]$, but the propensity to develop major bleeds after initiation of anticoagulation therapy remains unclear. Major life-threatening bleeding in critically ill COVID-19 patients remain not well described in terms of clinical characteristics, timing after the onset of the infection, initiation of anticoagulation, and affected anatomical sites.

In this study, we retrospectively observed the incidence of major bleeding in 25 critically ill COVID-19 patients admitted to the Intensive Care Unit (ICU) at the American University of Beirut Medical Center. Patients were predominantly males (68\%), with ages ranging from 15 to 88 years, and a length of stay (LOS) up to 125 days (mean LOS $=20$ days). Of those patients, 13 were on prophylactic anticoagulation using heparin or its derivatives, and 10 received a full dose of anticoagulation based on a valid medical indication (atrial fibrillation, myocardial infarction, limb ischemia, etc.). One patient received both anticoagulation and antiplatelet therapy due to an existing indication before COVID-19 infection and seven patients were receiving antiplatelet therapy prior to current illness and admission.

Out of the twenty-five patients evaluated in the study, six patients developed major bleed (24\%). Of these patients, four were on therapeutic anticoagulation at the onset of the bleed, representing $67 \%$ of the major bleeders, and one patient was on both anticoagulation and antiplatelet therapy. The characteristics, the uncommon bleeding sides, and the associated medical history of the patients with major bleeding are described.

\section{Case description}

The study included 25 critically ill COVID-19 patients who were predominantly male (68\%), with ages ranging from 15 to 88 years, and a length of stay (LOS) up to 125 days (mean $\mathrm{LOS}=20$ days). Of those patients, 13 were on prophylactic anticoagulation using heparin or its derivatives, and 10 received a full dose of anticoagulation based on a valid medical indication (atrial fibrillation, myocardial infarction, limb ischemia, etc.). One patient received both anticoagulation and antiplatelet therapy due to an existing indication before COVID-19 infection and seven patients were receiving antiplatelet therapy prior to current illness and admission.

Out of the twenty-five patients evaluated in the study, six patients developed major bleed (24\%). Of these patients, four were on therapeutic anticoagulation at the onset of the bleed, representing $67 \%$ of the major bleeders, and one patient was on both anticoagulation and antiplatelet therapy. The characteristics, the uncommon bleeding sides, and the associated medical history of the patients with the major bleed are described.

\section{Patient 1}

A 72-year-old man with multiple comorbid conditions was admitted to the COVID-19 ICU after suffering from acute respiratory distress syndrome (ARDS) secondary to COVID19 infection, requiring intubation and mechanical ventilation (MV). Before admission to ICU, the patient completed a course of azithromycin, and he then received two doses of convalescent plasma therapy. During his stay at COVID-19 ICU, the patient developed new-onset atrial fibrillation and therapeutic anticoagulation with heparin was initiated. His International Normalized Ratio (INR) was closely monitored and remained between 2 and 2.5. Eight days after the initiation of anticoagulation therapy, a significant drop in hemoglobin level was noted, and follow-up imaging demonstrated a large right gluteal and hamstring hematoma measuring $10 \times 6 \mathrm{~cm}$. Heparin was discontinued and the hematoma was treated conservatively with follow up studies demonstrating a progressive reduction in its size. Luckily, no embolic events were observed after discontinuing heparin, and the patient was transferred to a chronic respiratory care unit for chronic respiratory failure.

\section{Patient 2}

A 34-years-old male was diagnosed abroad with COVID-19 pneumonia requiring non-invasive ventilation. The patient was then transferred from another country after he received hydroxychloroquine and azithromycin for five days. Before transfer, he was also diagnosed with segmental pulmonary embolism and received a dose of Tenecteplase and followed by systemic therapeutic anticoagulation. Upon admission to our center, a brain imaging demonstrated multiple foci of micro hemorrhage in the subcortical white matter along with massive subarachnoid bleed, and a left cerebellar and right frontal stroke. After admission, the patient's condition continued to deteriorate with worsening ARDS, multiple bacterial, fungal infections, and eventually, he passed away.

\section{Patient 3}

A 66-year-old male, known to have hypertension (HTN), transferred to COVID-19 ICU with severe COVID-19 pulmonary involvement. The patient was started on intravenous (IV) dexamethasone $6 \mathrm{mg}$ daily for increased oxygen requirements, received a dose of tocilizumab, and ten days after admission, Remdesivir became available and the 
patient was started on it as well. Unfortunately, the patient continued to progress into severe ARDS that necessitated intubation and mechanical ventilation. His stay was also complicated by new-onset atrial fibrillation for which a therapeutic dose of enoxaparin was started. More than twenty days after the initiation of anticoagulation, a significant drop in the patient's hemoglobin level was noted with hemodynamic instability requiring blood transfusion. An abdominal computed tomography angiography (CTA) imaging noted active bleeding in the right gluteus medius muscle hematoma. The hematoma measured $9 \times 6 \mathrm{~cm}$. In addition, smaller right obturator internus muscle and right quadratus femoris muscle hematomas, with ongoing active bleeding that required multiple embolizations. After embolization and transfusion, the patient became stable and was transferred to the respiratory care unit for the difficulty to wean off mechanical ventilation.

\section{Patient 4}

A 50-year-old male, known to have HTN and chronic kidney disease (CKD), was admitted for COVID-19 ICU with respiratory failure requiring MV. He was started on tocilizumab, dexamethasone $6 \mathrm{mg}$ IV daily for 10 days, received two doses of tocilizumab. On the date of admission, 5000 units of heparin were administered subcutaneously three times daily for DVT prophylaxis. Nine days later, the patient suffered from massive and persistent hematuria that required multiple blood transfusions and frequent urinary bladder irrigation. The patient's condition continued to deteriorate, and he expired due to the progression of his ARDS, severe sepsis, and multiple organ failure.

\section{Patient 5}

An 82-year-old male, with a history of dementia, coronary artery disease (CAD) status post percutaneous transluminal coronary angioplasty (PTCA), on dual antiplatelet therapy, CKD, HTN, heart failure with a low ejection fraction of $25 \%$, was admitted for COVID-19 pneumonia. On admission, the patient was started on dexamethasone $6 \mathrm{mg}$ IV daily, a prophylactic dose of heparin 5000 units wad administered subcutaneously twice daily. One week after admission, the patient progressed into respiratory failure requiring intubation and MV. He was also noted to be hypotensive with an associated and a significant drop in Hemoglobin. Massive transfusion protocol was activated, and dual antiplatelets and heparin were immediately stopped. Further diagnostic and therapeutic interventions were abolished as the family requested comfort care and no further futile interventions. The patient subsequently passed away.

\section{Patient 6}

A 57-year-old female, with a history of asthma, HTN, and diabetes, was admitted with COVID-19 pneumonia. She was started on a prophylactic dose of enoxaparin and dexamethasone $6 \mathrm{mg}$ IV daily for 10 days. During her stay, the patient developed worsening and increasing oxygen requirements and was placed on a non-rebreather facemask with a $15 \mathrm{~L} /$ min flow of oxygen. A therapeutic dose of enoxaparin was then initiated and maintained for high clinical suspicion of pulmonary embolism. Six days after starting anticoagulation, the patient developed hypotension that was associated with a significant drop in $\mathrm{Hb}$ as well. CTA demonstrated left rectus abdominus hematoma and multiple retroperitoneal hematomas that were associated with ten, actively bleeding arteries. Artery embolization was needed on two occasions due to persistent hemorrhagic shock and recurrent bleeding. In addition, the patient suffered from significant hematuria. The active bleeding was eventually controlled, the patient stabilized and transferred to the chronic respiratory care unit.

\section{Discussion}

Our case series describes the excessive incidence of major bleeding in critically ill patients with COVID-19 infection placed on therapeutic anticoagulation with heparin or its derivative. We believe the incidence of bleeding is higher than what was previously reported, necessitating extra caution of treating physicians as to value the risk-benefit ratio of the anticoagulation in COVID-19 infected patients. Unfortunately, the elevated D dimer levels that is associated with COVID-19 infected patients is sometimes interpreted by treating physicians as an acute thromboembolism process, and hence the need for systemic anticoagulation (1). The increased incidence of major bleeding described in our study, suggests that further diagnostic studies and multidisciplinary clinical consensus are probably needed prior to initiating systemic anticoagulation. Moreover, the uncommon sites of bleeding noted in our sample suggest the need for aggressive and thorough evaluation, seeking to identify the site of bleeding and controlling it. Finally, further cohort studies of critically ill Covid-19 patients are clearly needed to identify the predisposing risk factors and mechanisms for identifying vulnerable subgroups and for better management of complications.

\section{Declarations}

Conflict of interest The authors declare no competing financial and non-financial interests. 
Research involving human subjects The reterospective study was reviewed and approved by the American University of Beirut Institutional Review Board (IRB) IRB ID \#: BIO-2020-0435.

\section{References}

1. Iba T, Levy JH, Levi M, Connors JM, Thachil J (2020) Coagulopathy of Coronavirus Disease 2019. Crit Care Med 48:1358-1364. https://doi.org/10.1097/CCM.0000000000004458

2. Klok FA, Kruip MJHA, van der Meer NJM, Arbous MS, Gommers D, Kant KM, Kaptein FHJ, van Paassen J, Stals MAM, Huisman MV, Endeman H (2020) Confirmation of the high cumulative incidence of thrombotic complications in critically ill ICU patients with COVID-19: an updated analysis. Thromb Res 191:148-150. https://doi.org/10.1016/j.thromres.2020.04.041

3. Al-Samkari H, Karp Leaf RS, Dzik WH, Carlson JCT, Fogerty AE, Waheed A, Goodarzi K, Bendapudi PK, Bornikova L, Gupta S, Leaf DE, Kuter DJ, Rosovsky RP (2020) COVID-19 and coagulation: bleeding and thrombotic manifestations of SARS-CoV-2 infection. Blood 136:489-500. https://doi.org/10.1182/blood. 2020006520

4. Dogra S, Jain R, Cao M, Bilaloglu S, Zagzag D, Hochman S, Lewis A, Melmed K, Hochman K, Horwitz L, Galetta S, Berger J (2020) Hemorrhagic stroke and anticoagulation in COVID-19. J Stroke Cerebrovasc Dis 29:104984. https://doi.org/10.1016/j.jstro kecerebrovasdis.2020.104984

5. Lucatelli P, De Rubeis G, Citone M, Lucarelli NM, Pasqualini V, Sturiale M, Giuliani S, Rosati M, Ceccherini C, Corona M, Mosconi C, Utili A, Argirò R (2020) Heparin-related major bleeding in Covid-19-positive patient: perspective from the outbreak. Cardiovasc Intervent Radiol 43:1216-1217. https://doi.org/ 10.1007/s00270-020-02532-3

6. Fraissé M, Logre E, Pajot $\mathrm{O}$, Mentec $\mathrm{H}$, Plantefève G, Contou D (2020) Thrombotic and hemorrhagic events in critically ill COVID-19 patients: a French monocenter retrospective study. Crit Care 24:275. https://doi.org/10.1186/s13054-020-03025-y

7. Schulman S, Kearon C (2005) Subcommittee on Control of Anticoagulation of the Scientific and Standardization Committee of the International Society on Thrombosis and Haemostasis. Definition of major bleeding in clinical investigations of antihemostatic medicinal products in non-surgical patients. J Thromb Haemost 3:692-4. https://doi.org/10.1111/j.1538-7836.2005.01204.x

8. Lauzier F, Arnold DM, Rabbat C, Heels-Ansdell D, Zarychanski R, Dodek P, Ashley BJ, Albert M, Khwaja K, Ostermann M, Skrobik Y, Fowler R, McIntyre L, Nates JL, Karachi T, Lopes RD, Zytaruk N, Finfer S, Crowther M, Cook D (2013) Risk factors and impact of major bleeding in critically ill patients receiving heparin thromboprophylaxis. Intensive Care Med 39:2135-2143. https:// doi.org/10.1007/s00134-013-3044-3

9. Shah A, Donovan K, McHugh A, Pandey M, Aaron L, Bradbury CA, Stanworth SJ, Alikhan et al (2020) Thrombotic and haemorrhagic complications in critically ill patients with COVID-19: a multicentre observational study. Crit Care 24:561. https://doi.org/ 10.1186/s13054-020-03260-3

Publisher's Note Springer Nature remains neutral with regard to jurisdictional claims in published maps and institutional affiliations. 